\author{
K. Mader • S. Kirchner • B. Wolfgarten • D. Pennig
}

\title{
Efficacy of the A-V Impulse System versus cryotherapy in the reduction of postoperative oedema of the hand: a prospective randomised trial
}

Received: 1 September 2006 / Accepted: 9 October 2006 / Published online: 14 December 2006

\begin{abstract}
In a prospective randomised trial, the effects of an intermittent compression hand pump vs. cryotherapy were compared on reduction of postoperative hand swelling and gain in finger movement after distal radius fractures. Although intermittent compression as a physical method for thromboprophylaxis and swelling reduction in orthopaedic and trauma patients of the lower leg are established, a prospective randomised trial for an objective evaluation of the effects of intermittent compression in the upper extremity has not been previously performed. Forty-three subjects $(63 \pm 33$ years, 32 women, 11 men $)$ with a unilateral distal radius fracture treated with transarticular external fixation
\end{abstract}

K. Mader $(\varangle) \cdot$ S. Kirchner $・$ B. Wolfgarten $\bullet$ D. Pennig

Department of Trauma and Orthopedic Surgery

Hand- and Reconstructive Surgery

St. Vinzenz-Hospital

Merheimer Str. 221-23, D-50733 Cologne, Germany

e-mail:k.mader@ndh.net were randomised into two treatment groups. In group A 21 patients were treated with cryotherapy. In group B 22 patients were treated with an intermittent compression hand pump. Reduction in swelling of the treated hand and MP and PIP joint movement were recorded with computerised assessment software (EVAL Hand Evaluation System) in comparison to the uninjured contralateral side. Reduction of swelling in group A was not statistically significant $(28.5 \%$ of total swelling or $0.61 \mathrm{~cm}$, SD $0.39, p=0.42)$, but in group $\mathrm{B}$ it was significant ( $92 \%$ of total swelling, $3.62 \mathrm{~cm}$, SD 1.48, $p<0.001)$. Comparison of increases in MP and PIP joint movement $(p<0.0016)$ showed statistically significant differences in favour of the intermittent compression pump. This study demonstrates that intermittent compression is more effective in the reduction of postoperative oedema and gain of finger movement of the hand than cryotherapy.

Key words Postoperative swelling - Intermittent compression pump • Cryotherapy $\cdot$ Hand $\cdot$ Distal radius fracture

\section{Introduction}

The wrist is a unique and highly complex joint which allows the hand to be positioned for the various demands of daily living. Acute swelling of the hand is a common problem after trauma or surgery and is associated with both pain and loss of function [1, 2]. Reduction of oedema is essential because swelling may interfere with blood supply and initiate algodystrophy. The therapeutic goal is to decrease soft tissue swelling, assist tendon mobility and prevent joint stiffness. Bulitta et al. [3] have demonstrated the benefit of using an intermittent compression device for reduction of swelling in the foot. Erdmann et al. [4] and Myerson and Henderson [5] have proved this in randomised trials. The results of Stranks et al. [6], using the A-V impulse system as a physical method of thromboprophylaxis in orthopaedic 
and trauma patients both with and without plaster immobilisation of the leg, indicated a benefit.

We compared the effects of a pneumatic compression device (A-V Impulse Hand Pump; Novamedix Services Ltd, Andover, UK) and cryotherapy in a prospective, randomised study to assess their efficacy in the reduction of postoperative oedema of the hand.

\section{Methods}

The aim of the study was to compare the relative efficacy of an intermittent compression system and cryotherapy in the reduction of postoperative oedema of the hand.

Between January 1996 and May 2000, 43 patients with unilateral fracture of the distal radius, and postoperative swelling of the hand of more than $3 \mathrm{~cm}$ in circumference, were enrolled in the study (Fig. 1). The a priori sample size estimation was 20 patients per group.

There were 11 men and 32 women with a mean age of 63 years (30-83 years)

\section{Inclusion criteria}

Patient entry criteria, as defined in the study protocol, were that the distal radius fracture was treated with a transarticular wrist fixator.

\section{Exclusion criteria}

Exclusion criteria were as follows:

bilateral fractures of the distal radius;

open fracture of the distal radius;

open injuries of the palm or dorsum of the hand;

ipsilateral fracture of the forearm or humerus;

ipsilateral fracture of the hand;

polytrauma;

amputation of the opposite limb.

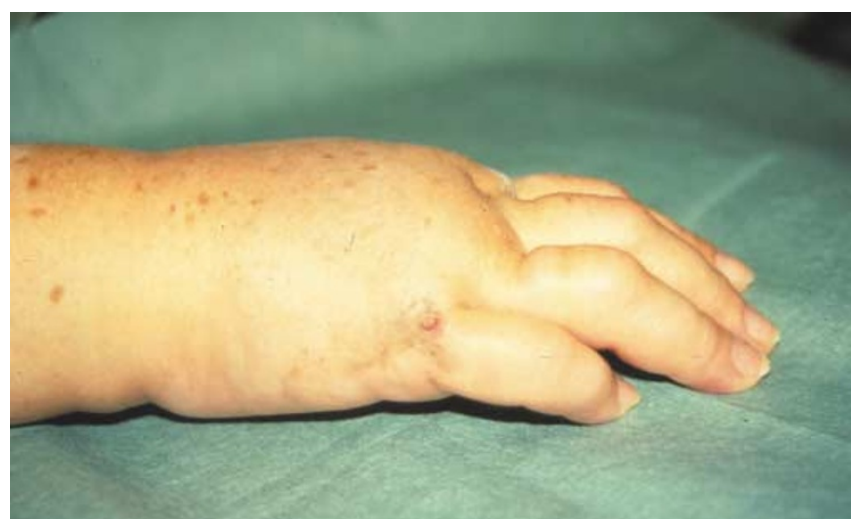

Fig. 1 Post-operative swelling of the right hand in a 66-year-old lady with distal radius fracture. The external fixator is applied radially and is not visible on this photograph due to the amount of swelling
Mode of treatment

All patients who met the inclusion criteria, were willing to participate in the trial and able to give informed consent were randomised into two groups, to receive either cryotherapy or the intermittent compression therapy.

Group A: Treatment of postoperative oedema of the hand with cryotherapy (cryotherapy group).

Group B: Treatment of postoperative oedema of the hand with the A-V impulse system (intermittent compression group).

\section{Group A}

The cryotherapy group comprised 21 patients. The average age of the 16 female and 5 male patients was 63 years (SD 14, range $30-81$ years). Each patient had the injured extremity stabilised preoperatively in a split plaster cast. The elevation of the limbs was $25^{\circ}$ by suspending the injured hand on a cushion. Preoperative therapy started at hospital admission and was continued until 7 days after operation. Cryotherapy was performed in this study with a 500-g ice-pack, which was applied with the following schedule: ten times per day for seven days, $20 \mathrm{~min}$ of continuous ice application. Subjects were asked to rest in a supine position with their forearm fully supinated and extended.

\section{Group B}

The intermittent compression group included 22 patients. The average age of the 16 female and 6 male patients was 66 years (SD 16, range 28-85 years). Each patient had the injured extremity stabilised preoperatively in a split plaster cast. In this group the A-V impulse system was used. This system is non-invasive and produces a high velocity surge of venous return. It consists of a bladder which is inflated to $130 \mathrm{mmHg}$ for $2 \mathrm{~s}$ every $20 \mathrm{~s}$ by an electrically driven air compressor. After wrapping the hand and wrist with a thin layer of stocking the device was placed on the palm, with the thumb located in the cut-out provided. The end of the cover was then wrapped tight across the back of the hand and secured by Velcro fastener (Fig. 2). The A-V impulse system is compatible with other treatments and therapies such as external fixation or casting. The pump was used in this group for seven days, five times per day for one hour.

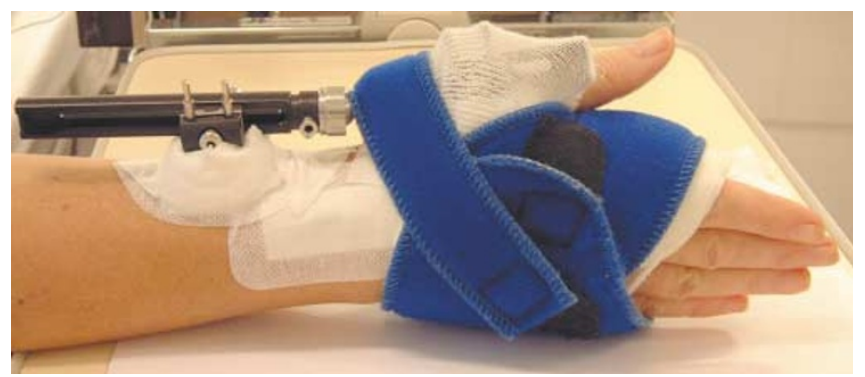

Fig. 2 The A-V impulse system is wrapped tightly across the back of the hand and secured by Velcro fastener. Due to the cut-out there is no impingement with the external fixator 
In both groups the state of elevation of the limb was $25^{\circ}$ starting from hospital admission until discharge during the whole therapy cycle. Immediately after surgery each patient of both groups started a similar physiotherapy regimen consisting of joint mobilisation, which was performed during intervals of either cryotherapy or intermittent compression device use.

\section{Data collection}

The initial hand circumference measurements were taken at the point of maximum swelling on the injured hand, and compared to those of the uninjured hand in exactly the same position. Both hands were marked with indelible ink so that subsequent measurements were carried out at identical points on each occasion. This measurement was repeated until the 7 th postoperative day.

Using the EVAL assessment software, MP and PIP joint movements of fingers 2-5 of both hands were measured. This software is a McIntosh compatible computer program, which allows reliable measurement of individual movements of finger joints. In addition, the EVAL assessment software is characterised by reproducible measurements. The measurement was performed pre- and postoperatively by the same investigator and

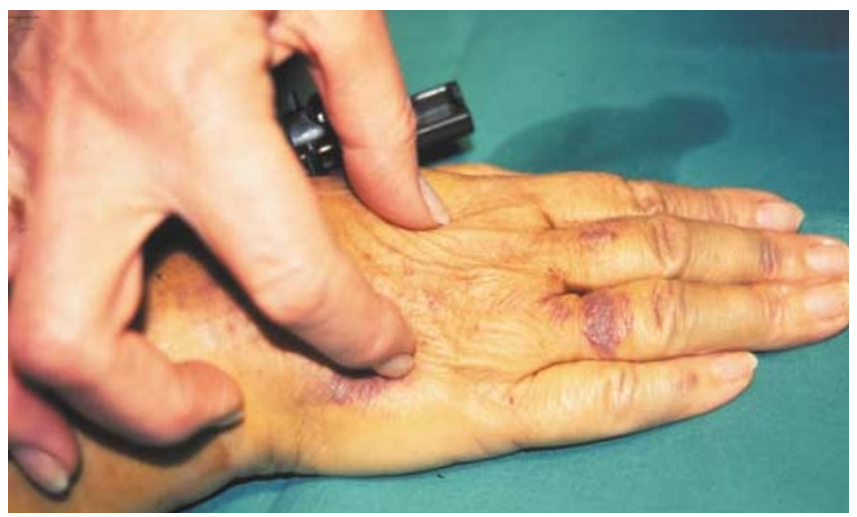

Fig. 3 Swelling reduction of the right hand in the same patient as in Figure 1 four days after intermittent compression pump usage. Full recovery of skin folds and soft tissue shape

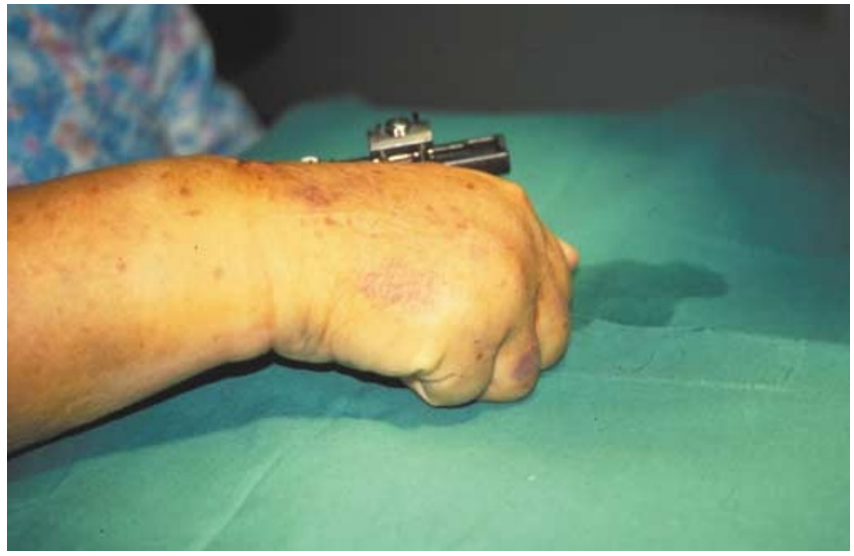

Fig. 4 Flexion of MP and PIP joints of the right hand of the same patient at the same time with regard to the treatment sessions every day. Measurement of hand circumference is an accepted and reliable tool, which is used in clinical follow-up, and for all reports of workers compensation insurance in Europe. The total duration of assessment was 7 days, when a final measurement was performed (Figs. 3 and 4).

\section{Statistical methods}

The distribution of the data was examined using the EVAL computer-aided assessment software (Hand Evaluation System), with an Apple McIntosh computer system. The results were expressed as a mean, with standard deviation. Between-group analysis was performed using the unpaired Student's $t$-test and within-group analysis by Student's $t$-test for paired observations. The circumference, MP and PIP joint movements of the injured and uninjured hands were indexed and the results expressed as median scores with standard deviations. For illustration, improvement of PIP and MP joint movement was plotted as index of total active motion (TAM). Between-group analysis was performed using the Mann-Whitney $U$-test and within-group analysis by the Wilcoxon signed rank test. The level of statistical significance was set at a $p$-value of 0.05 .

\section{Results}

In no case was there a complication of surgery that might have affected the course of recovery during the postoperative period. External fixation was used in all cases: minimally invasive percutaneous internal fixation in $80 \%$ in both groups and open reduction and internal fixation in $26 \%$ in group A and $23 \%$ in group B. Operative procedures were rated equal by an independent colleague.

\section{Cryotherapy group}

There was a reduction in swelling of $28.5 \%$ as measured at the point of maximum swelling on the injured and uninjured hands (Fig. 5). The mean reduction in hand circumference was $0.61 \mathrm{~cm}(\mathrm{SD} 0.39 ; p=0.42)$. The improvement in MP and PIP joint movement was not significant (Figs. 6 and $7, p=0.38)$.

\section{Intermittent compression group}

There was a reduction in swelling of $92 \%$ as measured at the point of maximum swelling on the injured and uninjured hands (Fig. 5). The mean reduction in hand circumference was $3.62 \mathrm{~cm}$ (SD 1.48; $p<0.001$ ). The improvement in MP and PIP joint movement was statistically significant (Figs. 6 and 7, $p<0.02$ ). 


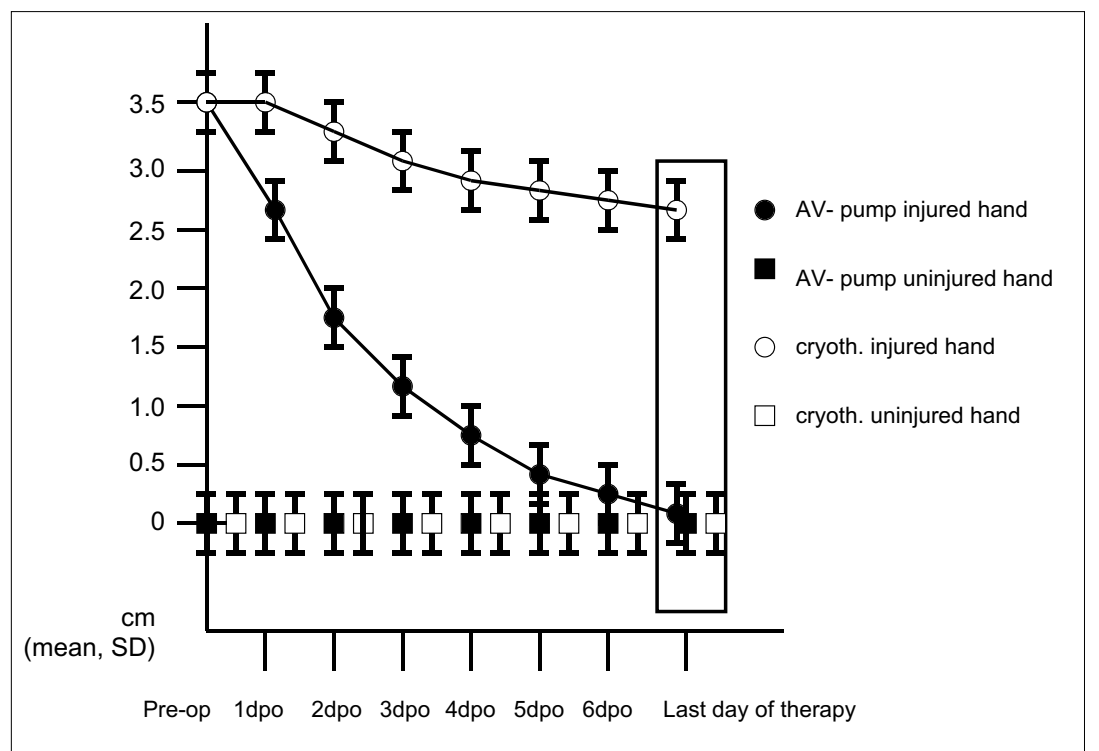

Fig. 5 Graphic illustration of swelling reduction during cryotherapy (Group A) and intermittent compression (Group B)

Fig. 6 Graphic illustration of improvement of PIP joint movement as index of TAM of the involved fingers without thumb

AV- pump

Cryotherapy

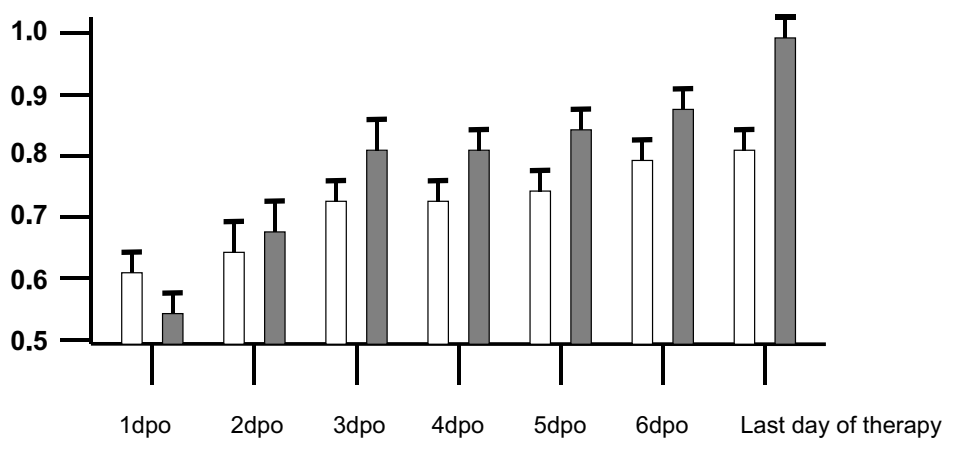

Index of TAM

MP

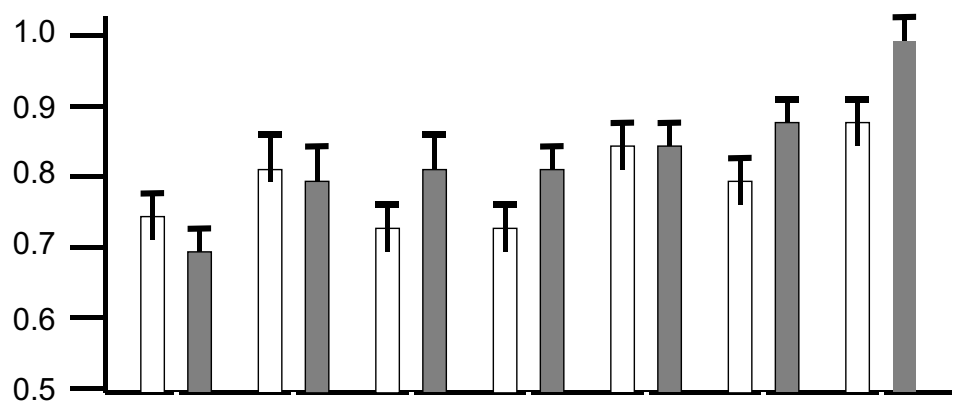

1dpo 2dpo 3dpo 4dpo 5dpo 6dpo Last day of therapy

Fig. 7 Graphic illustration of improvement of MP joint movement as index of TAM of the involved fingers without thumb 
There was a statistically significant difference in hand circumference between the two groups $(p<0.01$, Fig. 5) and for MP and PIP joint movement the Mann-Whitney $U$-test showed a statistically significant difference $(p<0.0016$, Figs. 6 and 7$)$ in both cases favouring the A-V impulse system.

\section{Discussion}

In 1996 Bulitta et al. [3] demonstrated a significant increase in venous blood return in the deep veins of the lower limb after applying a lower limb plaster cast with an intermittent compression device. Myerson and Henderson [5] showed a significant reduction of acute and chronic postsurgical swelling of the foot and ankle, using volumetric analysis. Erdmann et al. [4] demonstrated a significant reduction of pain, associated morbidity and convalescent time in intra-articular fractures of the calcis, a serious subset of trauma to the lower limb. Stranks et al. [6] showed that the incidence of deep venous thrombosis after hemiarthroplasty for hip fractures was reduced by increasing venous return with intermittent compression in the leg. In a prospective randomised study Asano et al. [7] demonstrated the preventative effect of using a foot pump on the incidence of pulmonary embolism. In a randomised trial Delis et al. [8] found that intermittent pneumatic foot compression appears to be effective in improving the walking ability and haemodynamics in patients with stable intermittent claudication.

These results indicate a benefit from using intermittent compression devices as a physical method of thromboprophylaxis in orthopaedic, trauma and vascular patients. Intermittent compression therapy was used in this randomised trial for the treatment of post-traumatic and postoperative oedema of the hand $[1,9]$.

Oedema indicates a malfunction of the circulatory system. Arterial pressure causes blood to flow to the arm, and a pumping mechanism is needed for the return flow of venous blood and lymphatic fluid [2]. Simons et al. [10] demonstrated three synergistic independent venous pumping systems in the hand: the superficial palmar, deep palmar and dorsal veins, which are activated by palm compression, isometric intrinsic muscle contraction and dorsal compression. Normal movements of the hand and arm provide the impetus to push the fluid towards the axilla, through numerous valves in the afferent vessels. Postoperatively, these normal movements cease or are inhibited, and the pneumatic compression device mimics the natural physiological process that maintains the blood circulation. Kilgour et al. [11] demonstrated that the forearm vascular resistance measured by electrical impedance plethysmography drops after local hand cooling during the first $30 \mathrm{~s}$ of cooling, and then gradually rises to reach peak levels between 5 and 6 min of cooling. This study shows that the A-V impulse system is significantly more effective than cryotherapy in the treatment of postoperative oedema of the hand. Berlin et al. [12] showed in a retrospective analysis of a large population the acute volume reduction in the affected hand and arm in postmastectomy lymphoedema.

Ramesh et al. [9] investigated the correlation between pneumatic compression of the hand and cubital vein blood flow. He found that continuous use of a pneumatic compression device (A-V impulse hand pump) for $48 \mathrm{~h}$ resulted in reduction in swelling of $78.6 \%$, and intermittent use a reduction of $69.4 \%$; they also found subjective reduction in pain perception and objective improvement in hand function. The reason for the improvement seen with the A-V impulse system could be an increase in the velocity of venous return as demonstrated by Duplex scanning of the medial cubital vein. In our pneumatic compression group we used the A-V impulse system four times per day for one hour, and this regime produced better results than cryotherapy. The reduction in swelling produces increased mobility of the MP and PIP joints, as we found in the EVAL assessment measurements. The increased range of motion of the uninvolved joints maintains mobility of joint capsule, ligaments and tendons, increases the circulation and assists in the reduction of oedema, as lack of mobility impairs outflow from the arm, causing increased oedema in the hand [2].

\section{Conclusions}

This study demonstrates that both reduction of postoperative swelling of the hand and gain in joint mobility are more significantly effected by using an intermittent compression hand pump than using cryotherapy. In our opinion the pneumatic compression device may be included in the therapeutic spectrum for postoperative oedema of the hand.

Acknowledgements One or more of the authors has received or will receive benefits for personal or professional use from a commercial party related directly or indirectly to the subject of this article. Funds were received in partial support of the research or clinical study presented in this article. The funding source was Novamedix Services Ltd, Andover, UK.

\section{References}

1. Moberg K (1960) The shoulder-hand-finger syndrome. Surg Clin North Am 40:367-372

2. Nicholas JS (1977) The swollen hand. Physiotherapy 63:285-286

3. Bulitta C, Kock HJ, Hanke J et al (1996) Förderung des venösen Rückstroms im Liegegipsverband durch das AV-Impulssystem. Unfallchirurgie 22:145-152 
4. Erdmann MWH, Richardson J, Templeton J (1992) Os calcis fractures: a randomized trial comparing conservative treatment with impulse compression of the foot. Injury 23:305-307

5. Myerson MS, Henderson MR (1993) Clinical applications of a pneumatic intermittent impulse compression device after trauma and major surgery to the foot and ankle. Foot Ankle 14:198-203

6. Stranks GJ, MacKenzie NA, Grover ML, Fail T (1992) The $\mathrm{A}-\mathrm{V}$ impulse system reduces deep vein thrombosis and swelling after hemiarthroplasty for hip fracture. J Bone Joint Surg Br 74:775-778

7. Asano H, Matsubara M, Suzuki K et al (2001) Prevention of pulmonary embolism by a foot sole pump. J Bone Joint Surg Br 83:1130-1132

8. Delis KT, Nicolaides AN, Wolfe JHN, Stansby G (2000)
Improving walking ability and ankle brachial pressure indices in symptomatic peripheral vascular disease with intermittent pneumatic foot compression: a prospective controlled study with one year follow up. J Vasc Surg 31:650-661

9. Ramesh M, Morrissey B, Healy JB et al (1999) Effectiveness of the A-V impulse hand pump. J Bone Joint Surg 81:229-233

10. Simons P, Coleridge-Smith P, Lees WR, McGrouther DA (1996) Venous pumps of the hand: their clinical importance. J Hand Surg Br 21:595-599

11. Kilgour RD, Carranza A, Findlay R (1997) Suppression of the reactive hyperaemic response in the forearm due to local hand cooling. Aviat Space Environ Med 68:46-50

12. Berlin E, Gjöres JE, Ivarsson C et al (1999) Postmastectomy lymphoedema: treatment and a five-year follow-up study. Int Angiol 18:294-298 